\title{
DNA with Artificial Base Pairs
}

\author{
Jürg Hunziker* and Gérald Mathis
}

\begin{abstract}
The introduction of a single base pair with the electronically complementary base surrogates phenyl- $(\mathbf{P})$ and pentafluorophenyl-deoxyriboside $\left(\mathbf{F}^{5}\right)$ into DNA oligonucleotides leads to a strong decrease in duplex stability. Longer stretches with alternating $\mathbf{P}-\mathbf{F}^{5}$ pairs can lead to duplexes with increased stability as compared to their counterparts with natural A-T base pairs. Optimization of the steric and electronic properties of the $\mathbf{P}-\mathbf{F}^{5}$ pair by replacing the phenyl residue with naphthalene, anisole or thioanisole leads to an increase in stability. Complementary charge distribution thus represents a novel design principle for artificial DNA base pairs. These results also highlight the importance of favorable intrastrand stacking interactions in the thermodynamic stabilization of oligonucleotide duplexes. A combination with favorable interstrand stacking could lead to a set of orthogonal, non-hydrogen bonded base pairs. Such artificial pairing systems could be used in many ways. By gradually changing the composition of linearly stacked artificial bases interesting electronic, photophysical and magnetic properties could result. The quasi one-dimensional arrangement of charge transfer complexes might pave the way for applications of the nucleic acid scaffold in material science.
\end{abstract}

Keywords: Artificial base pair $\cdot$ Charge transfer $\cdot$ DNA $\cdot$ Oligonucleotide $\cdot \pi$-Stacking

\section{Artificial Nucleic Acid Base Pairs}

The storage, replication, and translation of biological information are based on the specific formation of the hydrogen-bonded Watson-Crick A-T(U) and G-C base pairs. The generation of unnatural base pairs with thermal stability comparable to that of A$\mathbf{T}(\mathbf{U})$ and G-C but orthogonal coding capacity would significantly expand the biological and physicochemical potential of DNA and/or RNA. One might, for instance, envisage the incorporation of unnatural amino acids into proteins through the expansion of the genetic code, fluorescent labeling of nucleic acids, the use of universal bases in

\footnotetext{
${ }^{*}$ Correspondence: PD Dr. J. Hunziker Department of Chemistry and Biochemistry University of Bern

Freiestrasse 3

$\mathrm{CH}-3012$ Bern

Present Address:

Novartis Institutes for BioMedical Research

Genome and Proteome Sciences

WSJ-88.4.06

Postfach

$\mathrm{CH}-4002$ Basel

Tel.: +41613246102

E-Mail: juerg.hunziker@novartis.com
}

PCR primers or sequencing, or applications of the nucleic acid scaffold in material science.

In their pioneering work, Benner and coworkers [1-4] synthesized several unnatural nucleosides with altered hydrogen bonding schemes of the nucleobases (Fig. 1). They also successfully demonstrated that oligonucleotides containing such altered nucleobases can be replicated [2][5] as well as translated into proteins with unnatural amino acids in vitro [3]. At the same time, Rappaport investigated the Th-Gs base pair which resembles a C-G pair with only two hydrogen bonds. This system, however, suffers from interfering misinsertion of unmodified $\mathbf{C}$ opposite Gs during the replication with DNA polymerases [6]. More recent studies have utilized a combined strategy involving steric effects and hydrogen bonding groups. These $\mathbf{y}-\mathbf{x}$ and $\mathbf{y}$-s base pairs, which have been designed by Yokoyama et al., are enzymatically replicable as well [7-9]. A different approach toward altered hydrogen bond patterns was developed by Matsuda and co-workers [10][11]. They synthesized extended purine and pyrimidine base analogs with the capacity to form four hydrogen bonds (Fig. 1). Along similar lines, Kool and co-workers recently reported an artificial eight-base pairing system that relies on the regular Watson-Crick hydrogen bonding scheme but with steric restriction [12][13]. They demonstrated that the benzo-homologated forms of the canonical nucleobases, $\mathbf{x A}, \mathbf{x C}, \mathbf{x G}$, and $\mathbf{x T}$ (Fig.
1), pair with their respective natural counterparts thereby forming a right-handed duplex with a diameter approximately $2.4 \AA$ larger than that of regular DNA. Whether oligonucleotides in these latter two approaches are enzymatically replicable has not been investigated so far.

A different design strategy has been pursued in a number of laboratories wherein artificial DNA base pairs are held together by metal coordination instead of hydrogen bonds. Meggers et al. have designed a base pair with a pyridine-2,6-dicarboxylate nucleobase (Dipic) as a planar tridentate ligand, and a pyridine nucleobase (Py) as the complementary single donor ligand (Fig. 2) [14]. This system shows $\mathrm{Cu}^{2+}$-dependent pairing with the same level of stability as an A-T base pair. Another version of a metallo-base pair, the SPy-SPy base pair, which has higher stability than a G-C pair in the presence of one equivalent of $\mathrm{Ag}^{+}$and high selectivity, was later reported by the same group [15]. Shionoya and co-workers have recently reported a Ag(I)-mediated Py-Py base pair [16]. This base pair is more stable than an A-T pair but only in the presence of $\mathrm{Ag}^{+}$, not other metals. Similarly, Weizman and Tor introduced a 2,2'-bipyridine (Bpy) nucleoside inside a DNA duplex. The $T_{\mathrm{m}}$ of the Bpy-Bpy-containing DNA was the same as the corresponding natural oligonucleotides. Upon addition of 1 Equiv. of $\mathrm{Cu}^{2+}$ to the duplex, a significant increase in the $T_{\mathrm{m}}$ value was observed [17]. These stud- 


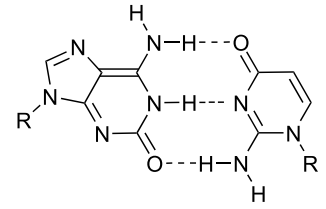

isoG<smiles></smiles>

$\mathbf{x}$

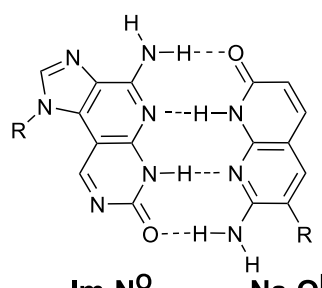

Im-N

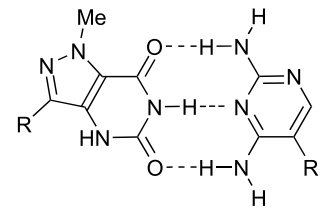

$\pi$

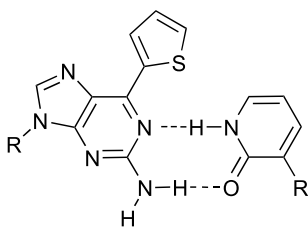

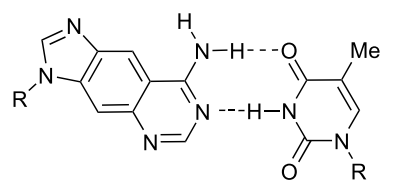

$\mathbf{x A}$

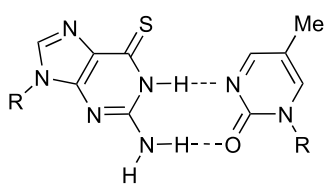

Gs

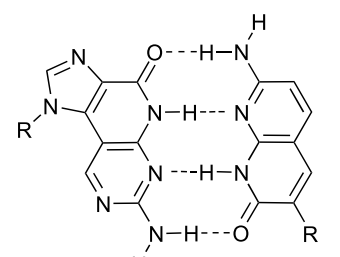

Im $-\mathrm{O}^{\mathrm{N}^{\mathrm{H}}}$

$\mathrm{Na}-\mathrm{N}^{\mathrm{O}}$

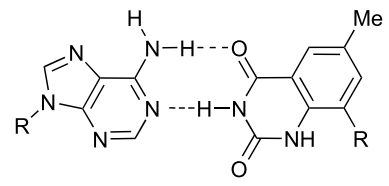

A

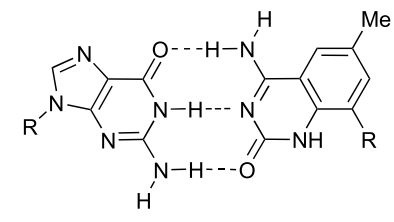

G
Fig. 1. Structures of unnatural base pairs with altered arrangement of hydrogen bond donors and acceptors. $R=2^{\prime}$-deoxyribofuranose.

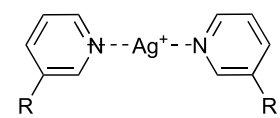

Py

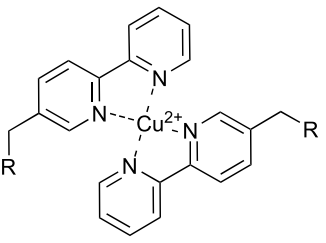

Bpy

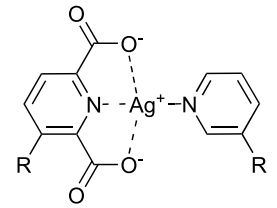

Dipic

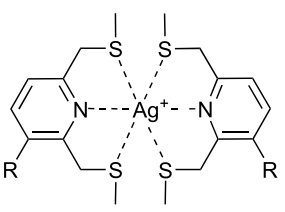

Spy

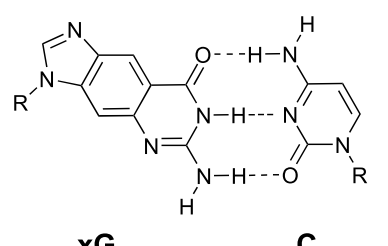

C

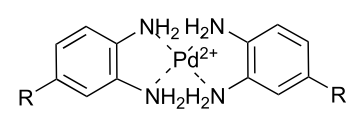

Phenylenediamine

ies demonstrate that metal coordination is typically stronger than hydrogen bonding but the corresponding duplexes can still thermally be denatured. Other nucleoside ligands in this category include aniline hydroxypyridone and porphyrins [18-22].

A third category constitutes hydrophobic base pairs which neither display complementary hydrogen bond patterns nor metal chelation. These new base pairs can stabilize DNA duplexes solely by shape complementarity and intra- or interstrand $\pi$-stacking. Surprisingly, they can still be replicable by DNA-polymerases in some cases. Such hydrophobic bases have been utilized as fluorescent probes or universal base analogs as well.

Kool described the first base pair between non-hydrogen bonding bases $\mathbf{Z}$ and F (Fig. 3), observing selectivity between these hydrophobic molecules [23]. This $\mathbf{Z}$-F base pair can be regarded as a nonpolar isoster of the A-T base pair. As such, the $\mathbf{F}$ base preferably leads to the incorporation of $\mathbf{A}$ in the opposite strand in a bacterial system [24]. Another example, developed by Matrey and Kool, is the pyrene and abasic site (Py-Ab) pair (Fig. 3) which can stabilize the DNA duplex by the base stacking and geometric fit alone [25]. Py-Ab is not a base pair in a strict sense since pyrene occupies the space of a full natural base pair. Nevertheless, it can be replicated by DNA polymerases [27]. Besides pyrene, deoxyribosides of other, unsubstituted aromatic systems, such as benzene, naphthalene, and phenanthrene, have been synthesized to study the influence of aglycon size on the stabilization of DNA duplexes by $\pi$-stacking [27]. Schul$\mathrm{tz}$, Romesberg and co-workers have also synthesized unnatural hydrophobic base pairs such as PICS-PICS (Fig. 3). Again, this base pair can be replicated by DNA polymerases with high selectivity although termination of polymerization is observed after the insertion of the unnatural base [28][29]. Another example which highlights the importance of $\pi$-stacking in the stabilization of DNA has been developed by Leumann and co-workers [30][31]. In these studies, the Bpy-Bpy base pair (Fig. 3) stabilizes the duplex more than natural base pairs mainly due to the stacking between the exterior pyridine rings of the Bpy residues. A Bpy-Ab pair is less stable than the Bpy-Bpy pair which has better aromatic stacking contacts. An unnatural hydrophobic base pair with shape complementarity between pyrrole-2-carbaldehyde (Pa) and 9-methylimidazol[(4,5)b]pyridine ( $\mathbf{Q})$ was recently developed by Yokoyama and co-workers (Fig. 3) [32]. This new Q-Pa base pair displays high specificity during enzymatic replication and leads to full length products.

Fig. 2. Structures of unnatural base pairs held together by metal-ligand interactions. $R=2^{\prime}$ deoxyribofuranose. 


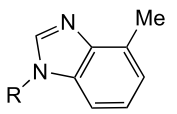

$\mathbf{Z}$

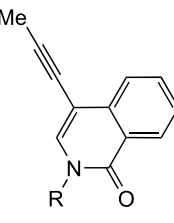

PICS

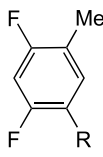

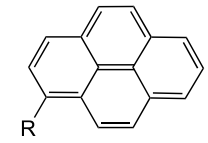

R

Py

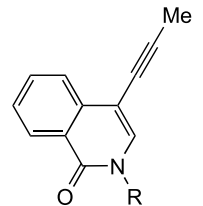

PICS
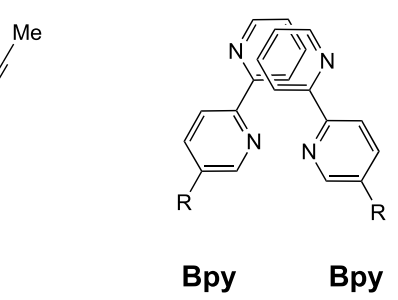

Fig. 3. Structures of unnatural base pairs relying on hydrophobic interactions for selective recognition. $\mathrm{R}=2^{\prime}$-deoxyribofuranose.

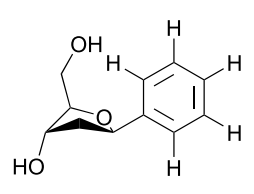

P

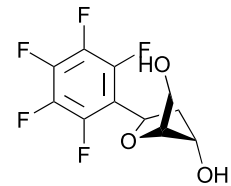

$F^{5}$

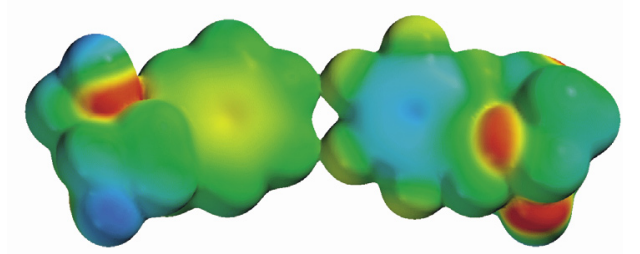

Fig. 4. The complementary charge distribution in the phenyl and pentafluorophenyl residues of the $2^{\prime}$-deoxyribosides $\mathbf{P}$ and $\mathbf{F}^{5}$ may lead to favorable edge-to-edge contacts between these two base surrogates similar to the arrangement of benzene and hexafluorobenzene molecules in their solid aggregate [33]

\section{Nucleic Acid Base Pairs Based on Charge Complementarity}

Based on the finding that hydrophobic effects can be exploited in addition to complementary hydrogen bonding to create novel base pairs summarized above, we proposed the incorporation of the pentafluorophenyl- and phenyl-C-deoxyribosides shown in Fig. 4 into synthetic oligodeoxynucleotides. In addition to purely hydrophobic interactions governing the selective mutual recognition of artificial nucleobases, a $\mathbf{F}^{\mathbf{5}}$-P base pair might be favored by the complementary charge distribution of the two aromatic rings. The consecutive alternating alignment of pentafluorophenyl and phenyl residues might result in very strong columnar $\pi$-stacking as seen in charge transfer complexes of mixtures of electron-rich and electron-poor aromatic compounds as displayed in the prototypic 1:1 aggregate formed by hexafluorobenzene and benzene [33][34].

Extending this design approach, we also synthesized the novel building blocks shown in Fig. 5. The larger aromatic residues present in these should on one hand increase the backbone distance since a $\mathbf{F}^{\mathbf{5}}$-P pair presumably has a shorter distance than that of a natural purine-pyrimidine base surrogates are more electron-rich than the original phenyl residue and should thus lead to enhanced stacking interactions in conjunction with the electron-poor pentafluorophenyl residue.

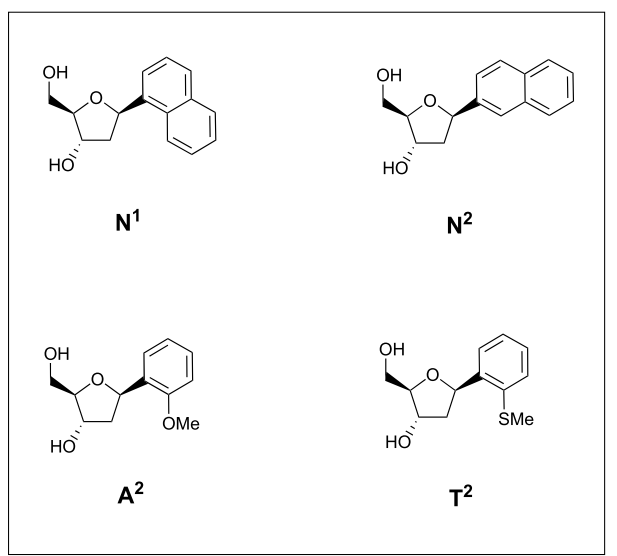

Fig. 5. Molecular structures of aromatic Cdeoxyribosides which have been prepared to investigate base stacking interactions with the pentafluorophenyl-deoxyriboside $\mathbf{F}^{5}$ pair. On the other hand, these new base
In order to analyze the stability of the new base pairs, several series of oligonucleotides have been synthesized in good efficiency by the phosphoramidite method. The ensuing pairing experiments showed that a single modified $\mathbf{F}^{\mathbf{5}}$-P base pair led to a strong decrease in duplex stability compared to the natural base pairs [35]. Nevertheless, when the phenyl base was replaced by naphthyl, anisyl or thioanisyl residues, a relative increase in duplex stability was observed. Moreover, the stability of the novel $\mathbf{F}^{\mathbf{5}}$-P base pairs is comparable to that of partially hydrogen-bonded base mismatches whereas the stability of $\mathbf{F}^{\mathbf{5}}-\mathbf{N}^{\mathbf{1}}, \mathbf{F}^{\mathbf{5}}-\mathbf{N}^{\mathbf{2}}, \mathbf{F}^{\mathbf{5}}-\mathbf{A}^{\mathbf{2}}$ and $\mathbf{F}^{\mathbf{5}}-\mathbf{T}^{\mathbf{2}}$ base pairs are comparable to that of the doubly hydrogen-bonded mismatches A-G and G-G as well as the wobble pair G-T [36]. These results highlight the influence of the size of the aromatic moiety opposite $\mathbf{F}^{\mathbf{5}}$, presumably through a reduction of sugar-phosphate backbone distortions, and the influence of electronic factors on intrastrand and interstrand base-base interactions.

Surprisingly, a self-complementary duplex containing four alternative $\mathbf{F}^{\mathbf{5}}$-P base pairs in a row was more stable than the natural counterpart with four A-T pairs. In this case, a duplex was formed at concentration above $3 \mu \mathrm{M}$. Below this threshold a monomolecular structure, presumably a hairpin, is predominant. The other self-complementary 10-mers duplexes preferred a monomolecular structure with constant $T_{\mathrm{m}}$ up to much higher concentration. To by-pass problems associated with self-complementarity, we have synthesized a series of nonself complementary 15-mers duplexes. The results of these experiments were that the duplexes containing the modified base are less stable than the duplexes with natural base pairs. However, the duplexes containing the $\mathbf{F}^{\mathbf{5}}-\mathbf{N}^{\mathbf{1}}$ and $\mathbf{F}^{\mathbf{5}}-\mathbf{N}^{\mathbf{2}}$ are more stable than the duplexes containing $\mathbf{F}^{\mathbf{5}}$-P base pairs. We also observed a decrease of $\Delta T_{\mathrm{m}}$ when the number of modified base pairs increased, which can be explained by the stabilization via favorable intrastrand $\pi$-stacking between aromatic residues of complementary electron density. However, no increase in $T_{\mathrm{m}}$ was observed when four consecutive or alternatingly arranged modified base pairs are introduced into the duplexes which seems to be a special case of the 10-mer self-complementary system.

The decrease in the stability of these duplexes containing one or several modified base pairs was addressed in a recent study by Kool and co-workers [37]. The authors argue that the pentafluorophenyl residue might induce a steric twist to the glycosidic bond which may distort the neighboring nucleobases as well. Indeed, duplexes with tetrafluorophenyl-C-deoxyribosides $\left(\mathbf{F}^{\mathbf{4}}\right)$ displayed increased stabilities. Further- 
more, dipole-dipole interactions - which are quite different for $\mathbf{F}^{\mathbf{4}}$ and $\mathbf{F}^{\mathbf{5}}$ - may be equally important for energetically favorable base-base contacts in addition to the dispersive van der Waals attraction.

In conclusion, complementary charge distribution as in the duplexes analyzed here represents a novel design principle for artificial base pairs. The results from this study highlight the importance of favorable intrastrand stacking interactions in the thermodynamic stabilization of oligonucleotide duplexes. These effects could still be optimized by using tetrafluorophenyl instead of pentafluorophenyl residues as the electrondeficient pairing partner. Moreover, the use of another non-hydrogen bonded base pair, $\mathbf{P y}-\mathbf{A b}$, in addition to the $\mathbf{P}-\mathbf{F}^{\mathbf{5}}$ pairs could aid in the development of DNA-like duplexes devoid of hydrogen bonds defining strand complementarity and holding the single strands together. Such an artificial pairing system could be used in many ways. By gradually changing the composition of linearly stacked artificial bases interesting electronic, photophysical and magnetic properties could result. The quasi one-dimensional arrangement of charge transfer complexes might pave the way for the application of the nucleic acid scaffold in material science.

\section{DNA with Artificial Bases in Molecular Electronics}

In 1974, Aviram and Ratner proposed a molecule in which a tetrathiofulvalene donor was linked covalently through a bridge to tetracyanoquinodimethide thus generating a molecular rectifier [38]. Since then a wealth of molecular electronic devices has been developed, e.g. molecular switches, memory elements, electrodes, solar cells, sensors, diodes. A particularly elaborate example of an artificial photosynthetic reaction center is shown in Fig. 6 [39].

It is evident from this example that synthesis becomes a limiting factor the more complex molecular electronic devices grow. In most cases the inherent difficulties associated with $\mathrm{C}-\mathrm{C}$ bond forming reactions preclude their use in the assembly of molecules with a molecular weight in excess of roughly 1000 Da. Even when changing to the most efficient covalent bond forming processes, the slow kinetics of large molecules put a limit to the degree of complexity achievable. - As in many other areas of chemistry, it is often useful to look at solutions to a particular problem provided by nature. Nucleic acids and proteins are formidable precedents for the organization of functional subunits into a larger supermolecule. Over the past 40 years, chemists have developed the most efficient procedures known for the synthesis of these classes of macromolecules. In solid-phase DNA synthesis repetitive coupling yields in excess of $99 \%$ are routinely achieved. It seems therefore fair to say that a system based on the oligonucleotide or peptide assembly strategy would make an ideal platform for sophisticated molecular electronic devices. The possibility to synthesize an oligomer chain one monomer at a time allows for the synthesis of spatially controlled assemblies with graded properties. In fact, $\alpha$-amino acids with electron donor and acceptor groups or porphyrin units attached to the sidechains have been assembled into peptide based molecular rectifiers or photonic wires [40][41].

A problem associated with the use of short peptide-like oligomers, however, is control of the secondary structure and hence the three-dimensional orientation of the electronically or photonically active substituents. A more rigid and preorganized scaffold would be needed. Double-stranded DNA is exactly such a system. The combination of two complementary single strands into a structurally uniform rod-like double helix allows for a persistence length of several dozen nanometers.

Effective charge transport has not only been demonstrated for conjugated organic polymers but also crystalline or discotic liquid crystalline systems composed of aromatic organic compounds [42][43]. Conductivity in these systems has been attributed to the formation of $\pi$-stacked columns of the aromatic residues and charge transport is most effective when the materials are doped to create mixed-valence states [44]. Such a $\pi$-stack is also present in doublehelical DNA. However, the notion that DNA may act as a molecular wire has recently been dismissed based on unequivocal experimental evidence [45]. Effective charge transport is only viable over very short distances. DNA behaves more like a large bandgap semiconductor [46]. Nevertheless, the idea that the $\pi$-stacked nucleobases constitute an electronically coupled ensemble with energy or charge transport capabilities is stimulating. Obviously, one would have to replace the hard to oxidize or reduce natural nucleobases with artificial ones which still undergo selective base-pairing.

Starting from the observed trend in the reduction potential of the natural bases $(\mathrm{T}<$ $\mathrm{C}<\mathrm{A}<\mathrm{G}$ ) the $\pi$-system of thymine could be extended and more electron-withdrawing substituents could be incorporated to lower its reduction potential further. Naphthalene diimide represents such an extended thymine (Fig. 7). It could undergo base-pairing with itself or with adenine and 2,6-diaminopurine. Whether the increased size of such base-pairs would still be compatible with natural deoxyribose-phosphate backbone remains to be seen. The example of xDNA mentioned above indicates that dsDNA may be more tolerant in this respect than previously thought. Alternatively, a pairing system with a much larger helical pitch, such as pRNA may be better suited [47].

Such a potential charge or energy carrier base moiety would need to be complemented by at least one other building block with differing electronic characteristics either to introduce directionality or to create alternating charge transfer base stacks. Partial realizations of such a system have recently been reported. Kool and co-workers created homo- as well as heterooligomers of deoxyribosides with different fluorescent base surrogates [48][49]. Through screening of a small library of bead-bound single-stranded tetramers with four different building blocks they could identify sequences with fluorescence intensities or emission wavelengths different from the monomeric units which make them up. Finally, Bouamaied and Stulz communicated an approach with porphyrins stacked in the major groove of DNA through covalent attachment to thymidine [50].

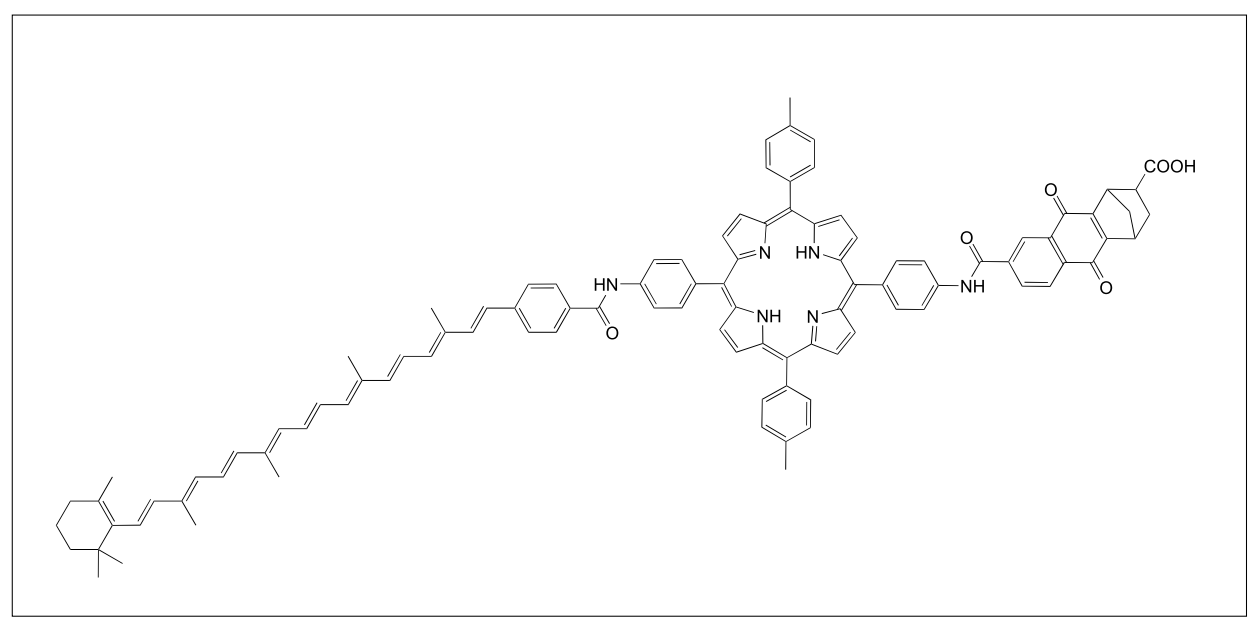

Fig. 6. This donor(1)-bridge-donor(2)-bridge-acceptor molecule acts as a mimic of photosynthetic solar energy transduction [39] 


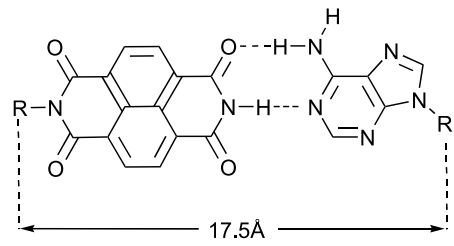

Naphthalimide

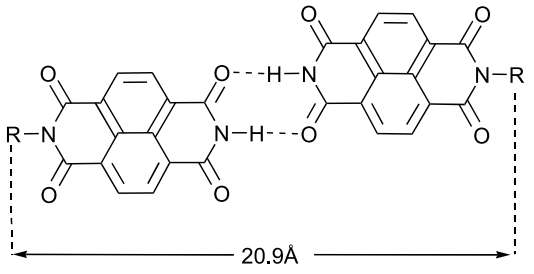

Naphthalimide Naphthalimide

Fig. 7. For the application of DNA in molecular electronic devices the hard to oxidize or reduce natural bases could be replaced by extended aromatic systems like naphthalene diimide which mimics the hydrogen bond pattern of thymine. The distances are obtained from semiempirical geometry optimization calculations. $\mathrm{R}=2$ '-deoxyribofuranosyl or ribopyranosyl residues.

- Without doubt, DNA will further prove its versatility in the assembly of sophisticated molecular electronic systems in the future.

Received: August 25, 2005

[1] C.Y. Switzer, S.E. Moroney, S.A. Benner, J. Am. Chem. Soc. 1989, 111, 8322.

[2] J.A. Piccirilli, T. Krauch, S.E. Moroney, S.A. Benner, Nature 1990, 343, 33.

[3] J.D. Bain, C. Switzer, A.R. Chamberlin, S.A. Benner, Nature 1992, 356, 537.

[4] J.J. Voegel, S.A. Benner, Helv. Chim. Acta 1996, 79, 1881.

[5] M.J.Lutz, J. Horlacher, S.A. Benner, Bioorg. Med. Chem. Lett. 1998, 8,1149.

[6] H.P. Rappaport, Biochemistry 1993, 32, 3047.

[7] T. Ohtsuki, M. Kimoto, M. Ishikawa, T. Mitsui, I. Hirao, S. Yokoyama, Proc. Natl. Acad. Sci. USA. 2001, 98, 4992.

[8] I. Hirao, T. Ohtsuki, T. Fujiwara, T. Mitsui, T. Yokogawa, T. Okuni, H. Nakayama, K. Takio, T. Yabuki, T. Kigawa, K. Kodama, T. Yokogawa, K. Nishikawa, S. Yokoyama, Nat. Biotechnol. 2002, 20, 177.

[9] M. Kimoto, M. Endo, T. Mitsui, T. Okuni, I. Hirao, S. Yokoyama, Chem. Biol. 2003, 11, 47.

[10] N. Minakawa, N. Kojima, S. Hikishima, T. Sasaki, A. Kiyosue, N. Atsumi, Y. Ueno, A. Matsuda, J. Am. Chem. Soc. 2003, 125, 9970.

[11] S. Hikishima, N. Minakawa, K. Kuramoto, Y. Fujisawa, M. Ogawa, A. Matsuda, Angew. Chem. 2005, 117, 602; Angew. Chem. Int. Ed. 2005, 44, 596.

[12] H. Liu, J. Gao, S.R. Lynch, Y.D. Saito, L. Maynard, E.T. Kool, Science 2003, 302, 868.

[13] J. Gao, H. Liu, E.T. Kool, Angew. Chem. 2005, 117, 3178; Angew. Chem. Int. Ed. 2005, 44, 3118.

[14] E. Meggers, P.L. Holland, W.B. Tolman, F.E. Romesberg, P.G. Schultz, J. Am. Chem. Soc. 2000, 122, 10714.

[15] N. Zimmermann, E. Meggers, P.G. Schultz, J. Am. Chem. Soc. 2002, 124, 13684.

[16] K. Tanaka, Y. Yamada, M. Shionoya, J. Am. Chem. Soc. 2002, 124, 8802.
[17] H. Weizmann, Y. Tor, J. Am. Chem. Soc. 2001, 123, 3375 .

[18] K. Tanaka, A. Tengiji, T. Kato, N. Toyama, M. Shiro, M. Shionoya, J. Am. Chem. Soc. 2002, 124, 12494.

[19] H. Morales-Rojas, E.T. Kool, Org. Lett. 2002, 4, 4377.

[20] K. Tanaka, M. Shionoya, J. Org. Chem. 1999, 64, 5002.

[21] M. Shionoya, K. Tanaka, Bull. Chem. Soc. Japan 2000, 73, 1945.

[22] K. Tanaka, M. Tasaka, H. Cao, M. Shionoya, Eur. J. Pharm. Sci. 2001, 13, 77.

[23] E.T. Kool, Acc. Chem. Res. 2002, 35, 936.

[24] J.C. Delaney, P.T. Henderson, S.A. Helquist, J.C. Morales, J.M. Essigmann, E.T. Kool, Proc. Natl. Acad. Sci. USA 2003, 100, 4469.

[25] T.J. Matray, E.T. Kool, J. Am. Chem. Soc 1998, 120, 6191 .

[26] T.J. Matray, E.T. Kool, Nature 1999, 399, 704.

[27] K.M. Guckian, B.A. Schweitzer, R.X.-F. Ren, C.J. Sheils, D.C. Tahmassebi, E.T. Kool, J. Am. Chem. Soc. 2000, 122, 2213.

[28] A.K. Ogawa, Y. Wu, D.L. McMinn, J. Liu, P.G. Schultz, F.E. Romesberg, J. Am. Chem. Soc. 2000, 122, 3274.

[29] E.L. Tae, Y. Wu, G. Xia, P.G. Schultz, F.E. Romesberg, J. Am. Chem. Soc. 2001, 123, 7439.

[30] C. Brotschi, A. Häberli, C. J. Leumann, Angew. Chem. 2001, 113, 3101; Angew. Chem. Int. Ed. 2001, 40, 3012.

[31] C. Brotschi, C.J. Leumann, Angew. Chem. 2003, 115, 1694; Angew. Chem. Int. Ed. 2003, 42, 1655.

[32] T. Mitsui, A. Kitamura, M. Kimoto, T. To, A. Sato, I. Hirao, S. Yokoyama, J. Am. Chem. Soc. 2003, 125, 5298.

[33] J.H. Williams, J.K. Cockcroft, A.N. Fitch, Angew. Chem. 1992, 104, 1666; Angew. Chem. Int. Ed. 1992, 31, 1655.

[34] C.R. Patrick, G.S. Prosser, Nature 1960 187, 1021.

[35] G. Mathis, J. Hunziker, Angew. Chem. 2002, 114, 3335; Angew. Chem. Int. Ed. 2002, 41, 3203.

[36] G. Mathis, 'Towards a DNA-Like Duplex Without Hydrogen-Bonded Base Pairs', Thesis, University of Bern, 2004.
[37] J.S. Lai, J. Qu, E.T. Kool, Angew. Chem. 2003, 115, 6155; Angew. Chem. Int. Ed. 2003, 42, 5973.

[38] J. Jortner, M. Ratner, 'Molecular Electronics', Blackwell Science, Oxford, 1997.

[39] D. Gust, T.A. Moore, A.L. Moore, Acc. Chem. Res. 2001, 34, 40.

[40] M.A. Fox, Acc. Chem. Res. 1992, 25, 569.

[41] N. Solladié, A. Hamel, M. Gross, Tetrahedron Lett. 2000, 41, 6075.

[42] T.J. Marks, Angew. Chem. 1990, 102, 886.

[43] C.W. Struijk, A.B. Sieval, J.E.J. Dakhorst, M. van Dijk, P. Kimkes, R.B.M. Koehorst, H. Donker, T.J. Schaafsma, S.J. Picken, A.M. van der Craats, J.M. Warman, H. Zuilhof, E.J.R. Sudhölter, J. Am. Chem. Soc. 2000, 122, 11057.

[44] L.L. Miller, K.R. Mann, Acc. Chem. Res. 1996, $29,417$.

[45] C. Wan, T. Fiebig, O. Schiemann, J.K. Barton, A. H. Zewail, Proc. Natl. Acad. Sci. USA 2000, 97, 14052.

[46] D. Porath, A. Bezryadin, S. de Vries, C. Dekker, Nature 2000, 403, 635.

[47] S. Pitsch, S. Wendeborn, R. Krishnamurthy, A. Holzner, M. Minton, M. Bolli, C. Miculca, N. Windhab, R. Micura, M. Stanek, B. Jaun, A. Eschenmoser, Helv. Chim. Acta 2003, 86, 4270.

[48] J. Gao, C. Strässler, D. Tahmassebi, E.T. Kool, J. Am. Chem. Soc. 2002, 124, 11590.

[49] A. Cuppoletti, Y. Cho, J.-S. Park, C. Strässler, E.T. Kool, Bioconjugate Chem. 2005, $16,528$.

[50] I. Bouamaied, E. Stulz, Chimia 2005, 59 , 101. 